\title{
The relationship between egg size and helper number in cooperative breeders: a meta-analysis across species
}

\author{
Tanmay Dixit ${ }^{\text {Corresp., }}{ }^{1}$, Sinead English ${ }^{1}$, Dieter Lukas ${ }^{1}$ \\ 1 Department of Zoology, University of Cambridge, Cambridge, United Kingdom \\ Corresponding Author: Tanmay Dixit \\ Email address: tanmay.dixit@cantab.net
}

Background. Life history theory predicts that mothers should adjust reproductive investment depending on benefits of current reproduction and costs of reduced future reproductive success. These costs and benefits may in turn depend on the breeding female's social environment. Cooperative breeders provide an ideal system to test whether changes in maternal investment are associated with the social conditions mothers experience. As alloparental helpers assist in offspring care, larger groups might reduce reproductive costs for mothers or alternatively indicate attractive conditions for reproduction. Thus, mothers may show reduced (load-lightening) or increased (differential allocation) reproductive investment in relation to group size. Given the growing number of studies which have investigated how cooperatively breeding mothers adjust pre-natal investment depending on group size, our aim was to assess whether in groups with more helpers mothers consistently show lower or higher pre-natal investment across species and to assess whether these changes relate to variation in post-natal investment.

Methods. We extracted data on the relationship between helper number and maternal pre-natal investment (egg size) from 12 studies on 10 species of cooperatively breeding vertebrates. We performed meta-analyses to calculate the overall estimated relationship between egg size and helper number, and to quantify variation among species. We also tested whether these relationships are stronger in species in which the addition of helpers is associated with significant changes in maternal and helper post-natal investment.

Results. Across studies, there is a significant negative relationship between helper number and egg size, suggesting that in most instances mothers show reduced reproductive investment in larger groups, in particular in species in which mothers also show a significant reduction in post-natal investment. However, even in this limited sample, substantial variation exists in the relationship between helper number and egg size, and the overall effect appears to be driven by a few well-studied species.

Discussion. Our results, albeit based on a small sample of studies and species, indicate that cooperatively breeding females tend to produce smaller eggs in larger groups. These findings on prenatal investment accord with previous studies showing similar load-lightening reductions in postnatal parental effort (leading to concealed helper effects), but do not provide empirical support for differential allocation. However, the considerable variation in effect size across studies suggests that maternal investment is mitigated by additional factors. Our findings indicate that variation in the social environment may influence life-history strategies and suggest that future studies investigating withinindividual changes in maternal investment in cooperative breeders offer a fruitful avenue to study the role of adaptive plasticity. 
1 The relationship between egg size and helper number in cooperative

2

3

4

$5{ }^{1}$ Department of Zoology, University of Cambridge, Downing Street, Cambridge, United

$\begin{array}{ll}6 & \text { Kingdom, CB2 3EJ } \\ 7 & \text { Corresponding Author: Tanmay Dixit }\end{array}$

$\begin{array}{ll}6 & \text { Kingdom, CB2 3EJ } \\ 7 & \text { Corresponding Author: Tanmay Dixit }\end{array}$

8 Email: tanmay.dixit@cantab.net

9

10

11

12

13

14

15

16

17

19

20

21

22

23

\section{Tanmay Dixit ${ }^{1}$, Sinead English ${ }^{1}$, Dieter Lukas ${ }^{1}$}

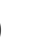

0

1

12

3

14

15

16

17

\section{8}

breeders: a meta-analysis across species 


\section{Summary}

Background. Life history theory predicts that mothers should adjust reproductive investment depending on benefits of current reproduction and costs of reduced future reproductive success. These costs and benefits may in turn depend on the breeding female's social environment. Cooperative breeders provide an ideal system to test whether changes in maternal investment are associated with the social conditions mothers experience. As alloparental helpers assist in offspring care, larger groups might reduce reproductive costs for mothers or alternatively indicate attractive conditions for reproduction. Thus, mothers may show reduced (loadlightening) or increased (differential allocation) reproductive investment in relation to group size. Given the growing number of studies which have investigated how cooperatively breeding mothers adjust pre-natal investment depending on group size, our aim was to assess whether in groups with more helpers mothers consistently show lower or higher pre-natal investment across species and to assess whether these changes relate to variation in post-natal investment.

Methods. We extracted data on the relationship between helper number and maternal pre-natal investment (egg size) from 12 studies on 10 species of cooperatively breeding vertebrates. We performed meta-analyses to calculate the overall estimated relationship between egg size and helper number, and to quantify variation among species. We also tested whether these relationships are stronger in species in which the addition of helpers is associated with significant changes in maternal and helper post-natal investment.

44 Results. Across studies, there is a significant negative relationship between helper number and egg size, suggesting that in most instances mothers show reduced reproductive investment in 
47 natal investment. However, even in this limited sample, substantial variation exists in the

48 relationship between helper number and egg size, and the overall effect appears to be driven by a

49 few well-studied species.

50 Discussion. Our results, albeit based on a small sample of studies and species, indicate that cooperatively breeding females tend to produce smaller eggs in larger groups. These findings on prenatal investment accord with previous studies showing similar load-lightening reductions in postnatal parental effort (leading to concealed helper effects), but do not provide empirical support for differential allocation. However, the considerable variation in effect size across studies suggests that maternal investment is mitigated by additional factors. Our findings indicate that variation in the social environment may influence life-history strategies and suggest that future studies investigating within-individual changes in maternal investment in cooperative breeders offer a fruitful avenue to study the role of adaptive plasticity.

Introduction

Life history theory suggests that, to maximize their lifetime reproductive success, breeders should adjust their reproductive investment according to both current and future conditions (Stearns, 1992). There is considerable evidence that increased maternal investment into offspring reproduction (Bolton, 1991; Lindström, 1999; Krist, 2011), but negatively influences maternal survival and investment into future reproductive attempts (Lack, 1947; Maynard Smith, 1977;

67 Berube, Festa-Bianchet \& Jorgenson, 1996; Metcalfe \& Monaghan, 2001; Lummaa \& CluttonBrock, 2002; Hanssen et al., 2005). A mounting body of studies has shown that mothers adaptively alter investment into reproduction via offspring traits, such as egg size or hormone 
70 provision into eggs, depending on breeding conditions, such as food availability or mate quality

71 and contribution to care (e.g. Cunningham \& Russell, 2000; Eising et al., 2001; Gil et al., 2004;

72 Bolund, Schielzeth \& Forstmeier, 2009). In addition, in group-living species, mothers might

73 adjust investment according to other salient features of their social environment besides their partner, in particular if there is variation in the presence or expected helping contribution of alloparental carers.

Cooperatively breeding species provide an opportunity to study the changes in parental investment in response to variability in the social group environment. In these species, nonbreeding helpers assist breeding individuals with caring for offspring (Stacey \& Koenig, 1990; Clutton-Brock, 2002; Koenig \& Dickinson, 2004). Differences in the number of additional group members, both within and among species, generate natural variation in the social environment mothers experience (Russell \& Lummaa 2009). Given the trade-offs between investment into the current brood and later reproductive attempts, investments by mothers into the current brood are expected to vary in the presence of helpers (Crick, 1992; Hatchwell, 1999; Savage, Russell \& Johnstone, 2013). Detailed field observations of various cooperative breeders, particularly in birds, have presented evidence that the amount of maternal investment at the nest (provisioning) is associated with variation in the workforce present when breeding (Hatchwell \& Russell, 1996; Woxvold, Mulder \& Magrath, 2006; Cockburn et al., 2008; Russell et al., 2008). Associations between maternal investment and helper numbers might result from differences in territory quality or maternal condition (Cockburn et al., 2008, Russell \& Lummaa, 2009). In at least some instances, however, reduced maternal provisioning may be interpreted as an adaptive responses of females to variation in the social group environment, such that breeding females maximize their lifetime fitness by reducing post-natal investment in current offspring when helpers 
93 compensate for such a reduction (Wright, 1998, Russell et al., 2008, McDonald, Kazem \&

94 Wright, 2009).

Social influences could also influence maternal pre-natal investment (Savage, Russell \&

96 Johnstone, 2015): in cooperative breeders, altered maternal investment into eggs might be compensated for or enhanced by the protection, provisioning or incubation of offspring provided by helpers subsequent to laying (Russell et al., 2007; Taborsky, Skubic \& Bruintjes, 2007;

Russell \& Lummaa, 2009). One hypothesis that has been proposed, the primary one we test here, is that cooperatively breeding mothers will show decreased investment into egg size when helpers are present to compensate for such reduced investment: the 'load-lightening hypothesis' (here onwards, LL hypothesis) (Russell \& Lummaa, 2009; Savage, Russell \& Johnstone, 2015; also sometimes referred to as 'concealed helper effects', Koenig, Walters \& Haydock, 2009). This hypothesis argues that, since egg production is costly in terms of future maternal survival and reproductive success (Visser \& Lessells, 2001; Williams, 2005), lifetime reproductive success will be increased if reductions in egg size have little or no effect on offspring fitness owing to the compensation provided by helpers (Savage, Russell \& Johnstone, 2013).

Observations that egg sizes decrease with an increased number of helpers at the nest would provide support for the LL hypothesis (Taborsky, Skubic \& Bruintjes, 2007; Russell \& Lummaa, 2009), though such an association could also reflect factors that influence pre- and post-natal conditions independently or changes in helper behaviour in response to maternal investment

112 (Savage, Russell \& Johnstone, 2015).

An alternative hypothesis is that more helpers can cause mothers to increase investment

114 to take advantage of good conditions: the so-called 'differential allocation (DA) hypothesis'

115 (Sheldon, 2000). Usually applied to when females have a particularly attractive mate (Sheldon, 
116 2000; Horvathova, Nakagawa \& Uller, 2011), it has been expanded to cooperative breeding,

117 predicting that if a female is assisted by a large numbers of helpers (indicating good breeding

118 conditions), she should increase investment due to higher current potential reproductive success

119 or the increased reproductive value of the current brood (Valencia et al., 2006; Russell \&

120 Lummaa, 2009, Savage, Russell \& Johnstone, 2015). Thus, the DA hypothesis, unlike the LL

121 hypothesis, would predict an increase in egg investment with increased number of helpers.

122 Variation in prenatal maternal investment is likely to be associated with the care

123 offspring receive post-natally (Savage, Russell \& Johnstone, 2015). Here we focus on two

124 factors that might shape the relationship between prenatal maternal investment and postnatal

125 offspring care: mothers are expected to show reduced investment in egg size if their reduced

126 investment can be directly substituted by helper care (helpers provide food, and helper efforts

127 can buffer a small size early in life) and if there are no benefits to offspring of receiving

128 investment beyond a certain threshold (for example if offspring need to reach a certain body size

129 to increase their survival, but additional increments in size do not lead to higher offspring

130 fitness). The interactions between mothers and helpers during the post-natal stage might provide

131 information on these two factors. First, if prenatal investment can be substituted by postnatal

132 care, we would predict that mothers show consistent reductions in their pre- and postnatal

133 investment whereas if prenatal investment primes offspring to benefit from increased postnatal

134 help, maternal postnatal care behaviour should be independent of the presence of helpers.

135 Accordingly, we predict that mothers are more likely to produce smaller eggs in groups with

136 helpers in species in which mothers also reduce their postnatal investment in the presence of

137 helpers. Second, if additional investments continue to provide benefits, helping efforts are 
139 beneficial, helping efforts might plateau. In this case, we predict that mothers are more likely to

140 produce smaller eggs in larger groups in species where total helping efforts plateau and do not

141 increase linearly with group size.

142 In this study, we perform a meta-analysis to determine whether the currently existing

143 evidence indicates that variation in maternal prenatal investment in the form of egg size

144 systematically varies with the number of helpers across cooperative breeders, and whether the 145 direction of the relationship indicates whether load-lightening or differential allocation might be 146 more prevalent. Our study systematically combines the recently available observations to assess

147 whether consistent increases or decreases in maternal investment at the egg stage in response to

148 helper numbers have been observed and test predictions that changes in maternal pre-natal

149 investment are associated with the post-natal care offspring experience.

150

151 Materials \& Methods

152 A literature search was conducted to find relevant papers in which egg investment traits and

153 helper number were measured. We focused on egg size as our measure of maternal investment as

154 it is clearly under maternal control while subsequent investment into offspring is frequently

155 shared among mothers, fathers, and helpers. As such, we decided not to include data on

156 mammals since offspring size at birth is not available for most cooperatively breeding mammals

157 and lactation makes it more difficult to ascertain when investment is fully under maternal

158 influence versus that of helpers. For the purpose of this analysis, cooperative breeders were

159 defined as species where additional group members act as allomaternal helpers and provide

160 resources that can replace and hence compensate for adjustments in maternal investment, such

161 that mothers could potentially alter their investment into egg size based on helper number. 
162 Studies providing a quantitative measure of the link between maternal investment and group size

163 in eusocial species are rare. Moreover, we did not specifically search for these as our focus was

164 on instances where maternal investment could vary both pre- and post-natally: in eusocial

165 species, mothers tend not to provide any offspring care beyond egg production when helpers are

166 present and almost all post-natal care is performed exclusively by helpers (Keller and Chapuisat,

167 2010). We also did not include species where offspring care is shared only among reproductively

168 active females (e.g., Grinsted, Breuker \& Bilder, 2014), as it is difficult to determine how

169 individual mothers differ in their investment. On the other hand, cooperatively polyandrous

170 species were included: while additional group members (in this case, males) may share paternity,

171 efforts by the males represent alloparental care and could replace female investment into the

172 provisioning and protection of offspring (Davies, 1985).

173 Six relevant papers (Legge, 2000; Taborsky, Skubic \& Bruintjes, 2007; Koenig, Walters

174 \& Haydock, 2009; Canestrari, Marcos \& Baglione, 2011; Santos \& Macedo, 2011; Lejeune et

175 al., 2016) were located using keyword searches on Google Scholar with the search terms 'egg

176 size' and 'helpers', on 30th October 2016 (search carried out by TD and confirmed by DL; search

177 criteria were specific and hence disagreements were solved easily by discussion). Forward and

178 backward citation searches were then performed to find five further relevant studies (Russell et

179 al., 2007; Paquet et al., 2013; Santos \& Nakagawa, 2013; Santos, 2016; Valencia et al., 2016).

180 One study (Langmore et al., 2016) was identified as an 'online-early' abstract during the literature

181 search, and was included as full publication occurred during the course of our study. Relevant

182 studies were defined as those that investigated a relationship between egg size - measured

183 specifically as egg mass or egg volume - and number of helpers (determined by reading abstracts

184 and figures). Studies were then included if they provided the required test-statistic for use in 
185

186

187

meta-analysis (see below), or authors were contacted for raw data if suitable data were not provided in the paper. Where a direct measure of egg volume was not available, this was calculated from length and width using Hoyt's formula (Hoyt, 1979).

In seven studies, the change in maternal investment per helper was not provided, rather comparisons were given between investment when breeding in groups versus pairs. In these cases, and in cases where data on egg size traits with helpers were referred to but not provided, authors were contacted to provide the raw data for number of helpers in each group, and linear models, using the command lme in the package 'nlme' (Pinheiro et al., 2015) in RStudio version 0.99486 (RStudio team, 2015) using R version 3.2.2 (R Core Team 2015), were used to calculate test statistics. The test statistics $-F, t$ and $\chi^{2}-$ were converted to the statistic ' $r$ ' using formulae provided by Rosenthal (1994), Lajeunesse (in Koricheva et al., 2013), and Rosenberg (2010). Standard formulae were then Z-transformed, controlling for the asymptotic behaviour of the statistic $r$. We followed the detailed advice in Jennions, Moller \& Petrie (2001) for these methods, and formulae therein for calculations of effect sizes, transformations, and variances. We also investigated whether any relationship between maternal pre-natal investment and group size varied according to how aspects of post-natal care depend on helper number (specifically, whether mothers reduce post-natal care in the presence of helpers, and whether helpers provide benefits to offspring additively or up to a threshold). We thus searched for additional data on each species on the relationship between maternal post-natal care, helper effects on offspring and group size. Specifically, for each data point, we recorded whether the amount of support mothers provide to offspring (in most studies measured as the number of feeds per hour) is significantly lower when at least one helper (of that sex, in instances where the original study provided separate information for each sex on the relationship between egg size 
208 and helper number) is present than when groups only consist of mothers and their mate. To

209 determine whether helping effort increases linearly or plateaus with group size (all studies we

210 included showed either of these two options), we checked which function authors had used to

211 model the relationship between group size and total amount of care provided at the nest

212 (measured as number of feeds per hour in bird species, and as egg caring in Neolamprologus

213 pulcher), inspected the associated graphs to see whether increases of a second, third, etc. helper

214 was similar to the increase observed in groups with just a single helper. In addition, we read

215 through the associated paper carefully to check if the description by the authors confirmed the

216 graphical pattern, for example if they stated that the amount of support increased linearly with

217 group size.

218 Functions in the R package 'metafor' (Viechtbauer, 2010) were used to compute

219 heterogeneity and average effect sizes, and to create funnel plots to visualise the data. Cochran's

220 Q-test was used to test heterogeneity between effect sizes in all analyses carried out.

221 Heterogeneity describes the variation in the data which can be attributed to actual variation

222 between species, variation in study design, or publication bias (Nakagawa \& Santos, 2012). The

223 average effect size describes the overall effect of helpers on changes in maternal investment into

224 eggs, and hence indicates the strength and direction of the effect, determining whether the LL or

225 DA hypothesis receives more empirical support. Contour-enhanced funnel plots were used to

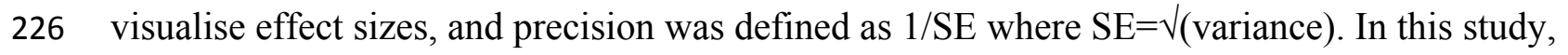

227 with high heterogeneity predicted, a contour plot would suggest a lack of publication bias if the

228 outliers occurred at all precision values, and not solely at low precision (Macaskill, Walter \&

229 Irwig, 2001). Publication bias was also quantified using Egger's regression test (Egger et al.,

230 1997) on the dataset, using the regtest function in the metafor package, and sample standard error 
231 as a predictor (Viechtbauer, 2010). We followed suggested best practices to minimize bias in the

232 dataset: the literature search was comprehensive, authors were contacted if published statistics

233 were insufficient to compute effect sizes, and the analysis included a study (Russell et al., 2007)

234 even though it did not contain the information required to compute the effect size in exactly the

235 same manner as the other effect sizes (Gates, 2002). However, our sample remains small, with

236 high heterogeneity since authors might have tested hypotheses with opposite effects (negative for

237 LL and positive for DA), such that we are aware that any conclusions are likely to remain limited 238 at this stage.

239 A multilevel linear model with restricted maximum likelihood estimation (REML)

240 analysis was carried out on all effect sizes, controlling for study as a random factor, to take into

241 account the non-independence of individual effect sizes calculated from the same study. In

242 several of the studies, authors reported multiple relationships: running separate analyses on male

243 and female helpers, reporting data on both egg size and mass, using different statistical models,

244 considering variation among or within individual females, or considering different mating

245 systems. Each study focussed on a single species, and in all but two cases, studies considered

246 different species. The exceptions were Paquet et al. (2013) and Santos (2016), both of which

247 studied sociable weavers; and Russell et al. (2007) and Langmore et al. (2016), both of which

248 studied superb fairy-wrens. As all of the studies in our sample had slightly different

249 methodologies, we initially controlled for study rather than species as a random term as we

250 deemed this a more parsimonious measure for data non-independence. We also conducted a

251 separate analysis controlling for species. Given the small sample size (10 species), we decided

252 not to include phylogenetic relatedness among species as a potential covariate. 

with the relationship between pre-natal maternal investment and group size, additional analyses

255

were carried out on all effect sizes, using the type of behaviour ('mothers decrease postnatal investment (in groups with on helper compared to pairs)' versus 'mothers do not decrease postnatal investment (no change or increase when a helper is present); 'helper benefits increase linearly (with group size)' versus 'helper benefits plateau') as a moderator (i.e. predictive variable). Separate multilevel linear mixed effects models, with REML analyses, were conducted for each post-natal response trait (maternal response and helping effort pattern), controlling for species or for study. Following these analyses, in order to more intuitively examine the difference among species, we carried out separate multilevel linear models with REML analysis, controlling for species, on the subset of the data where mothers reduce postnatal care, on the subset of the data where mothers do not reduce postnatal care, on the subset of the data where helping efforts increase linearly with group size, and on the subset of the data where helping efforts plateau with group size, in order to examine in more detail the difference in effect sizes.

\section{Results}

20 effect sizes from 12 studies of 10 species (9 bird species; 1 fish species) were extracted from publications or calculated from raw data (see table S1). There was no evidence of publication bias using Egger's regression test (test value $Z=-1.03 ; p=0.30$ ). In addition, the funnel plot was approximately symmetrical (figure 1), particularly for low values of 1/SE (i.e. low precision), suggesting little publication bias. As visible on the funnel plot (figure 1), the overall effect size (when controlling for study) was negative and significantly different from zero (effect size = - 
$2760.182, p=0.02$, upper and lower confidence limits $=-0.024$ and $-0.340 ; n=20$ effect sizes from

27712 studies). The Cochran's Q-test value was highly significant ( $Q=128$ on 19 degrees of

278 freedom, $\mathrm{p}<0.001$ ), suggesting that the data are highly heterogeneous. When the same dataset

279 was analysed controlling for species rather than study, the effect size was negative and

280 marginally not significant (effect size $=-0.164, \mathrm{p}=0.08$, upper and lower confidence limits $=$

2810.020 and $-0.348 ; n=20$ effect sizes from 10 species).

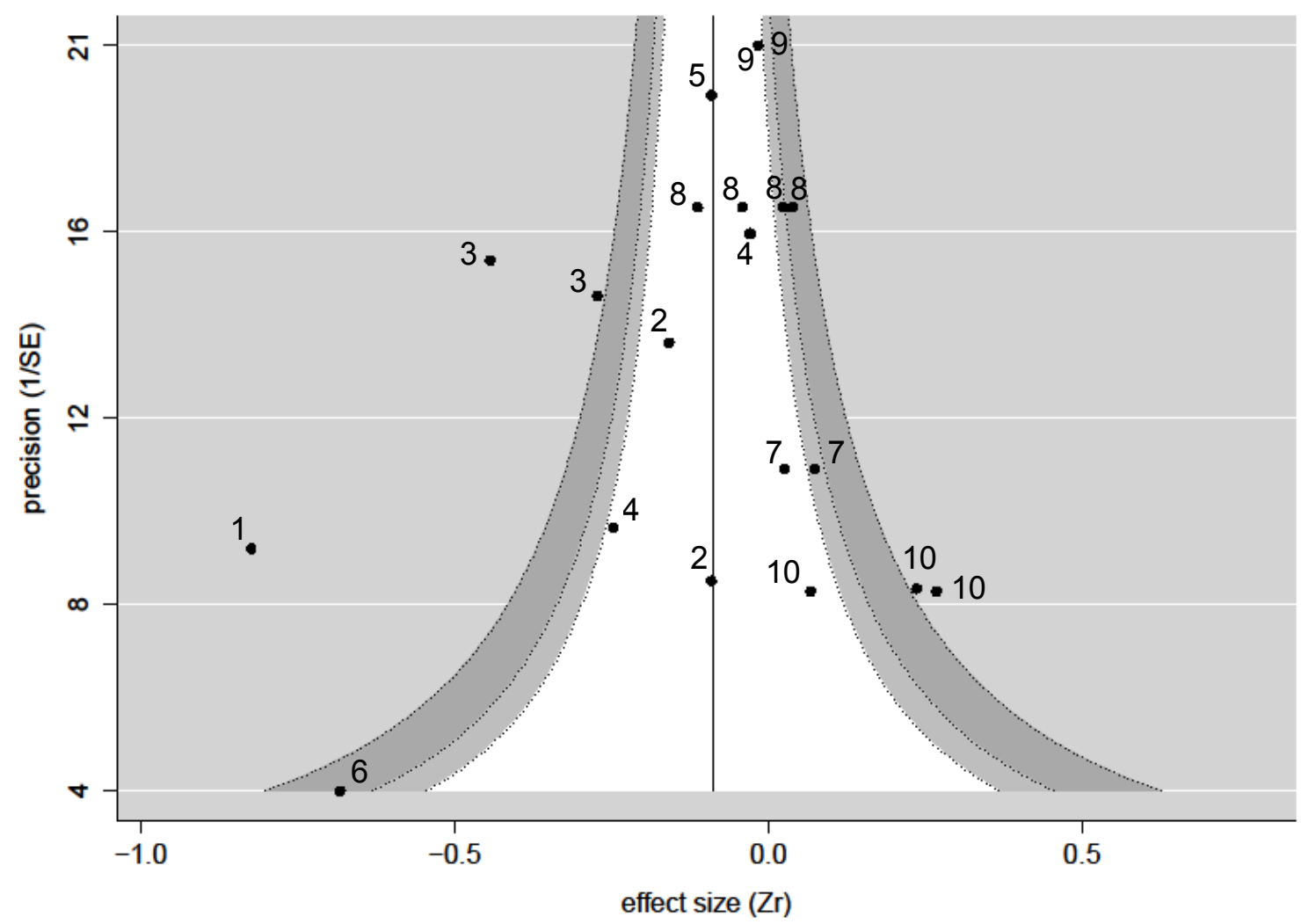

282

283

284

285

286

287

Figure 1: A funnel plot showing the 20 effect sizes extracted from 12 studies on 10 species. The pooled estimate (solid line), controlling for study, is significantly smaller than zero. The funnel shows the regions where 90\% (white), 95\% (light-grey), and 99\% (dark-grey) of values are expected to fall - there are several values outside of the funnel, and the heterogeneity is significant. Numbers next to values refer to species identity (1: Corvus corone; 2: Melanerpes formicivorus; 3: Philetairus socius; 4: Malurus cyaneus; 5: Vanellus chilensis; 6: Neolamprogus 
289

290

291

292

293

294

295

296

297

298

299

300

301

302

303

304

305

306

307

308

309

310

pulcher; 7: Prunella modularis; 8: Malurus elegans; 9: Cyanopica cooki; 10: Dacelo

novaguinea).

When we examined how the relationship between pre-natal investment (egg size) and helper number varied depending on how mothers adjust post-natal investment in larger groups, we found that - in analyses controlling for study - egg sizes are significantly more likely to be reduced in the presence of more helpers in species in which mothers also reduce their post-natal investment in the presence of helpers (estimate ['mothers decreases postnatal investment in larger groups' versus 'mothers do not decrease postnatal investment'] $=-0.336 \pm 0.158, p=0.03$, upper and lower confidence limits $=-0.026$ and $-0.645 ; \mathrm{n}=20$ effect sizes from 12 studies). A similar, but non-significant, relationship between post-natal maternal response and the relationship between egg size and helper number is found when controlling for species rather than study (estimate [reduction in postnatal investment present versus not present] $=-0.337 \pm 0.178, \mathrm{p}=$ 0.06, upper and lower confidence limits $=-0.012$ and $0.685 \mathrm{n}=20$ effect sizes from 10 species). The overall effect size (i.e. of the relationship between helper number and egg size) for those studies where mothers reduce their post-natal investment in the presence of helpers was significantly less than zero controlling for species identity (effect size $=-0.275, p=0.02$, upper and lower confidence limits $=-0.046$ and $-0.503, \mathrm{n}=13$ effect sizes from 7 species) (figure $2 \mathrm{a}$ ), with significant heterogeneity among values $(Q=98.5$ on 12 degrees of freedom, $\mathrm{p}<0.001)$. There are only very few investigations of effects where mothers do not reduce their post-natal investment in the presence of helpers, restricted to three species, but the overall effect size for these is close to zero (effect size $=0.062, \mathrm{p}=0.33$, upper and lower confidence limits $=0.187$ 
311 and $-0.064, \mathrm{n}=7$ effect sizes from 3 species) (figure $2 \mathrm{~b}$ ), with very low heterogeneity $(\mathrm{Q}=8.85$

312 on 6 degrees of freedom, $\mathrm{p}=0.18$ ).

313

314 The effects of helper presence on egg size do not appear to systematically differ between

315 instances in which helper efforts increase linearly with group size and those in which helping

316 efforts plateau (estimate ['helping effort increases linearly with group size' versus 'helping effort

317 plateaus'] $=-0.057 \pm 0.057, \mathrm{p}=0.31$, upper and lower confidence limits $=0.054$ and $-0.168 ; \mathrm{n}=19$

318 effect sizes from 11 studies - we excluded on study in which the relationship between helper

319 number and helper effort was unknown). The same analysis controlling for species similarly

320 shows no influence of how helping changes with group size on the relationship between egg size

321 and helper numbers (estimate [helping increase linear vs plateau] $=-0.052 \pm 0.057, \mathrm{p}=0.37$,

322 upper and lower confidence limits $=0.060$ and $-0.164 \mathrm{n}=19$ effect sizes from 9 species). 


\section{a) mothers decrease postnatal investment}

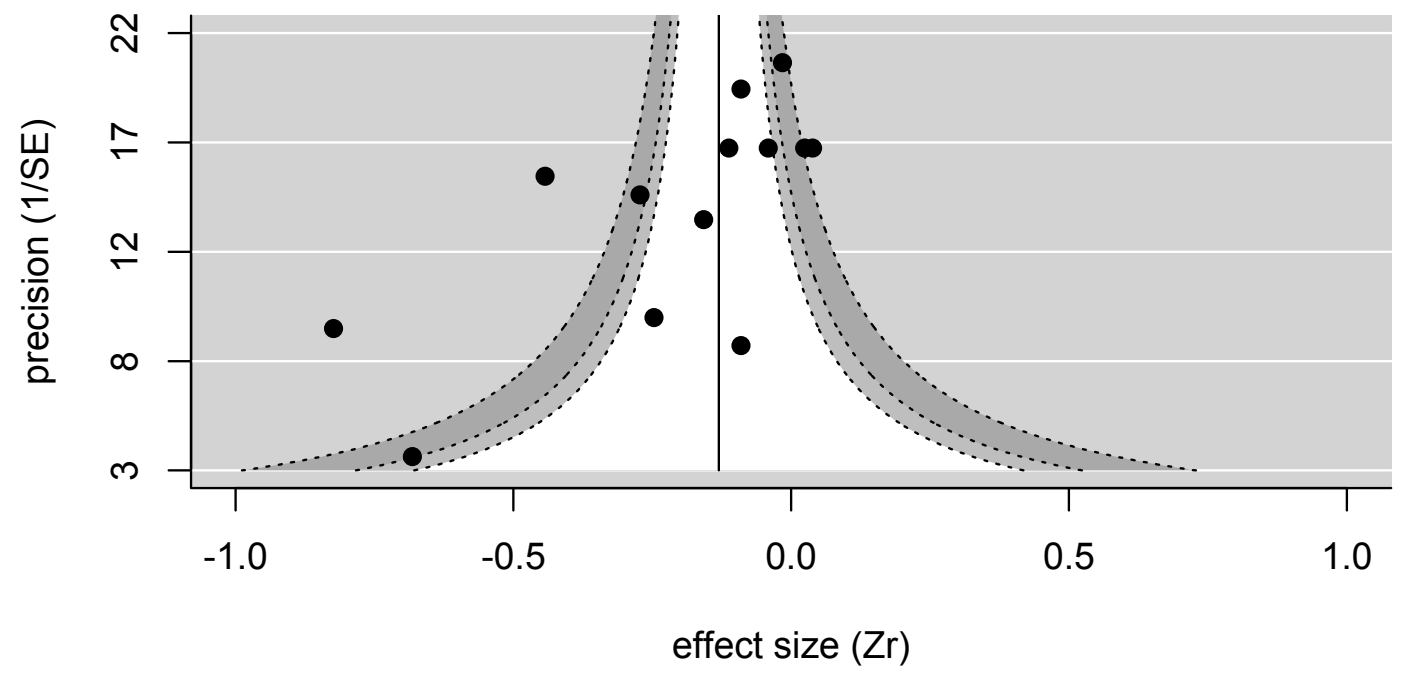

b) mothers do not decrease postnatal investment

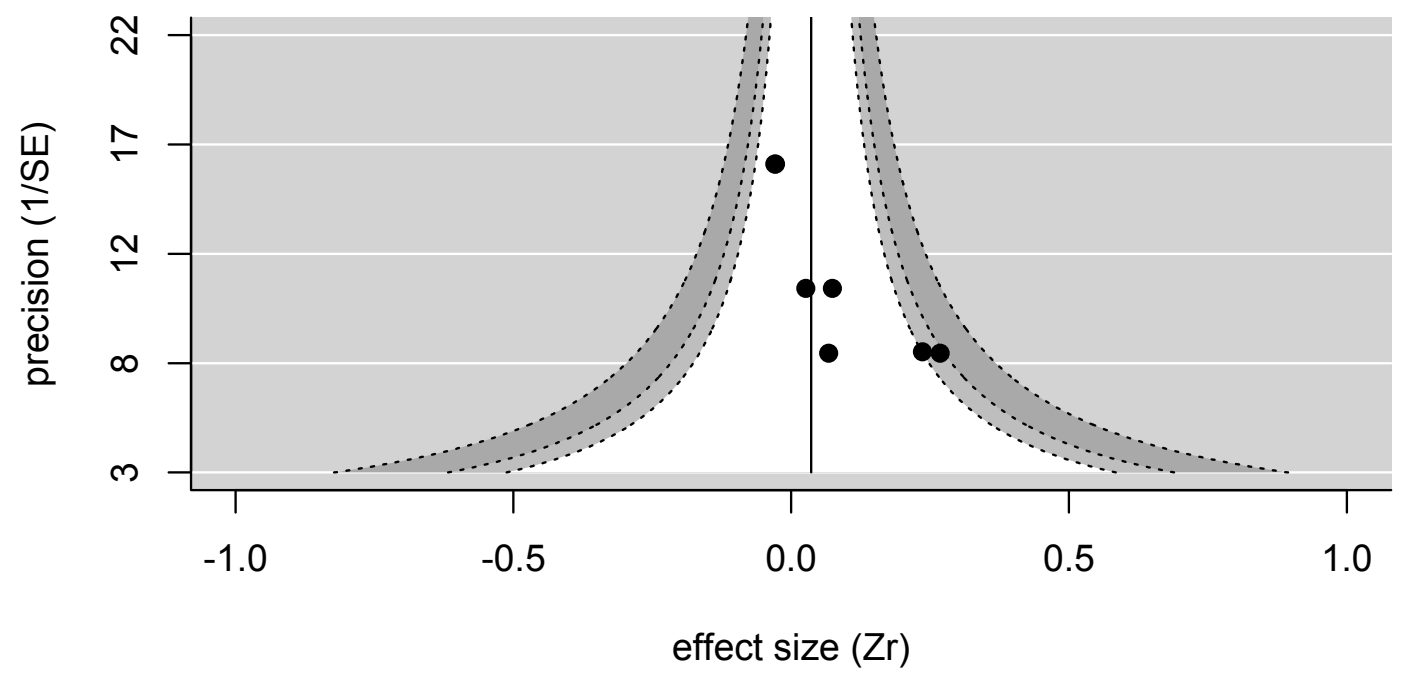

324

325 Figure 2: Funnel plots showing the distribution of effect sizes in species where (a) mothers

326 reduce postnatal investment in the presence of helpers (13 effect sizes from 10 studies in 7

327 species) or (b) mothers do not reduce postnatal investment in the presence of helpers (7 effect

328 sizes from 3 studies in 3 species). Solid line and shaded areas indicate pooled effect size and

329 expected values as explained in Figure 1. For instances where mothers reduce postnatal

330 investment (a), the pooled estimate (controlling for species) is significantly smaller than zero,

331 with significant remaining heterogeneity. For instances where mothers do not change or even 
332 increase their postnatal investment in the presence of helpers (b), the pooled estimate (controlling

333 for species) is not different from zero, with no significant heterogeneity among effect sizes.

Discussion

Our results suggest that in most studies which have examined how investment by

337

338

339

340

341

342

343

344

345

346

347

348

349

350

351

352

353

breeding females varies with group size, results show that females produce smaller eggs if more helpers are present. We also find that the association between smaller eggs and more helpers is particularly pronounced when females show reduced post-natal care in the presence of helpers, while egg sizes do not appear to vary with helper number when females do not adjust, or even increase, their level of offspring care in the presence of helpers. These findings suggest that concealed helper effects might occur in cooperative breeders, whereby helper efforts compensate for reduced investment by breeding females and thus provide them opportunities for loadlightening. We note that, given the small sample size and associated low statistical power, the overall effect we detected is weak and that even among these few species there is large unexplained variation in the association between maternal investment and the presence of helpers. Moreover, only a minority of studies specifically focused on within-female adjustments ( 3 of the 20 analyses in our dataset), and hence we cannot conclusively infer whether maternal responses are an example of adaptive plasticity rather than among-female differences. Changes in egg size could be due to other factors, such as greater intra-group competition, although in the few detailed studies such explanations have been ruled out (e.g. Canestrari, Marcos \& Baglione 2011). In spite of these limitations, we explain below the insights to be gained from our study for our understanding of the benefits of helpers to breeding individuals. 
Our findings are based on a small sample of correlative studies, most of which compared

355

356

357

358

359

360

361

362

363

364

365

366

367

368

conditions across, rather than within, individual females. There are multiple, potentially non-

exclusive explanations for why, in most species, egg sizes are smaller in nests that are tended for

by a large number of alloparental carers. For example, it has previously been suggested that competition among females (Russell \& Lummaa 2009) or the relative costs of pre- versus postnatal care (Savage, Russell \& Johnstone, 2015) might influence patterns of maternal investment. However, our results do appear to reject the prediction of the differential allocation hypothesis, as females do not appear to increase investment in the presence of helpers. Instead, female investment - both pre- and postnatal - appears to be consistently reduced in groups with more helpers, which supports the prediction of the load-lightening hypothesis. The prevalence of effects consistent with load-lightening in our dataset could explain the common observation that helper effects on offspring size or fledging success are often weak or absent, as care efforts by helpers might be directly counterbalanced by a decrease in maternal investment (i.e. loadlightening), hence leading to little difference in offspring traits in groups with or without helpers (Russell et al., 2007; Cockburn et al., 2008; Koenig, Walters \& Haydock. 2009; Paquet et al., 2015). The evidence existing thus far indicates that adaptive plasticity in maternal investment in response to helper number is at least possible. Further detailed studies focusing on variation within individuals offer the opportunity to assess the role of pre-natal maternal investment in understanding selection on cooperative systems (Taborsky, Skubic \& Bruintjes, 2007; Russell \& Lummaa, 2009). The scope of comparative studies could be increased by not only focusing on quantitative changes in egg size, but by also including other evidence of helper effects, such as indications of load-lightening in eusocial insects including bees (Shpigler et al., 2013), ants (Villet, 1990), and termites (Matsuura \& Kobayashi, 2010). 
We observed large variation across species in the effect of helper numbers on egg size.

Differences among species might reflect that in some instances changes in female investment

reflect non-adaptive processes, but even in cases where plasticity in egg size results from

adaptive processes, the direction and magnitude of changes in maternal investment likely reflect

maternal investment with a greater number of helpers (i.e. favourable conditions) is reproductive

lifespan, with increased investment in favourable conditions in species with short lifespan and

decreased investment in favourable conditions in long-lived species (Paquet et al. 2015). The

occurrence of LL or DA may also depend on environmental conditions (Hatchwell, 1999,

Langmore et al. 2016) - in poor conditions, females should increase investment when helpers are

present in order to gain some reproductive success, because the marginal impact of care has a

relatively large influence on offspring fitness. In good conditions, the impact of small amounts of

additional care is low, and hence mothers would be selected to reduce care in the current

survival of offspring or reproductive success beyond that which would be expected from

provisioning alone (for example by reducing predation risk), breeders may be selected to

increase investment in the presence of helpers (Carranza et al., 2008), due to the high

reproductive value of the current brood in comparison with potential future broods (Valencia et

primarily apply when variation in maternal pre-natal investment is in the size of each offspring,

and different relationships might occur if changes in maternal investment are associated with the 
399 small offspring rather than the same number of larger offspring, more help is likely to be

400 beneficial (e.g. Liebl et al., 2016).

401 Our findings support the idea that adaptive plasticity in reproduction, with females

402 reducing investment in current broods to prioritise future survival and reproduction, might occur

403 in response to changes in the social environment. Further studies are needed to test whether

404 effects hold consistently on a within-female level and whether increases in fecundity and

405 survival are seen when load-lightening occurs. Identifying the conditions favouring the evolution

406 of plasticity in reproductive investment has wider implications, as in many animal species the

407 social environment is more variable than the physical environment and, indeed, social factors

408 often mediate physical environmental factors (e.g. through foraging competition).

409

410

411

412

413

414

415

416

417

418

420

421

\section{6}

419 Arnold, K. E., \& Owens, I. P. (1998). Cooperative breeding in birds: a comparative test of the

\section{Acknowledgements}

Many thanks to all the authors who responded to requests for raw data: Walt Koenig, Naomi Langmore, Sarah Legge, Eduardo Santos, and Juliana Valencia. We thank Xavier Harrison, Andrew Russell and one anonymous reviewer for constructive comments on earlier versions of this manuscript.

\section{References} life history hypothesis. Proceedings of the Royal Society of London B: Biological Sciences, 265(1398), 739-745. 
422 Berube, C. H., Festa-Bianchet, M., \& Jorgenson, J. T. (1996). Reproductive costs of sons and 423 daughters in Rocky Mountain bighorn sheep. Behavioral Ecology, 7(1), 60-68.

424 Bolton, M. (1991). Determinants of chick survival in the lesser black-backed gull: relative 425 contributions of egg size and parental quality. The Journal of Animal Ecology, 949-960.

426 Bolund, E., Schielzeth, H., \& Forstmeier, W. (2009). Compensatory investment in zebra finches:

427 females lay larger eggs when paired to sexually unattractive males. Proceedings of the Royal 428 Society of London B: Biological Sciences, 276(1657), 707-715.

429 Brown, J. L., (1987). Helping and Communal Breeding in Birds: Ecology and Evolution. 430 Princeton University Press, Princeton.

431 Burley, N. (1986). Sexual selection for aesthetic traits in species with biparental care. American 432 Naturalist, 415-445.

433 Burley, N. (1988). The differential-allocation hypothesis: an experimental test. American 434 Naturalist, 611-628.

435 Canestrari, D., Chiarati, E., Marcos, J. M., Ekman, J., \& Baglione, V. (2008). Helpers but not 436 breeders adjust provisioning effort to year-round territory resource availability in carrion 437 crows. Animal Behaviour, 76(3), 943-949.

438 Canestrari, D., Marcos, J. M., \& Baglione, V. (2011). Helpers at the nest compensate for reduced 439 maternal investment in egg size in carrion crows. Journal of evolutionary biology, 24(9), 18704401878. 
441 Carranza, J., Polo, V., Valencia, J., Mateos, C., \& de la Cruz, C. (2008). How should breeders

442 react when aided by helpers? Animal Behaviour, 75(4), 1535-1542.

443 Clutton-Brock, T. (2002). Breeding together: kin selection and mutualism in cooperative

444 vertebrates. Science, 296(5565), 69-72.

445 Cockburn, A., Sims, R. A., Osmond, H. L., Green, D. J., Double, M. C., \& Mulder, R. A. (2008).

446 Can we measure the benefits of help in cooperatively breeding birds: the case of superb

447 fairy-wrens Malurus cyaneus? Journal of Animal Ecology, 77(3), 430-438.

448 Crick, H. Q. (1992). Load-lightening in cooperatively breeding birds and the cost of

449 reproduction. Ibis, 134(1), 56-61.

450 Cunningham, E. J., \& Russell, A. F. (2000). Egg investment is influenced by male attractiveness

451 in the mallard. Nature, 404(6773), 74-77.

452 Davies, N. B. (1985). Cooperation and conflict among dunnocks, Prunella modularis, in a

453 variable mating system. Animal Behaviour, 33(2), 628-648.

454 Egger, M., Smith, G. D., Schneider, M., \& Minder, C. (1997). Bias in meta-analysis detected by 455 a simple, graphical test. $B m j, 315(7109), 629-634$.

456 Eising, C. M., Eikenaar, C., Schwabl, H., \& Groothuis, T. G. (2001). Maternal androgens in 457 black-headed gull (Larus ridibundus) eggs: consequences for chick development. Proceedings of 458 the Royal Society of London B: Biological Sciences, 268(1469), 839-846.

459 Gates, S. (2002). Review of methodology of quantitative reviews using meta-analysis in 460 ecology. Journal of Animal Ecology, 71(4), 547-557. 
461 Gil, D., Leboucher, G., Lacroix, A., Cue, R., \& Kreutzer, M. (2004). Female canaries produce

462 eggs with greater amounts of testosterone when exposed to preferred male song. Hormones and 463 Behavior, 45(1), 64-70.

464 Grinsted, L., Breuker, C. J., \& Bilde, T. (2014). Cooperative breeding favors maternal 465 investment in size over number of eggs in spiders. Evolution, 68(7), 1961-1973.

466 Hanssen, S. A., Hasselquist, D., Folstad, I., \& Erikstad, K. E. (2005). Cost of reproduction in a 467 long-lived bird: incubation effort reduces immune function and future reproduction. Proceedings 468 of the Royal Society of London B: Biological Sciences, 272(1567), 1039-1046.

469 Hatchwell, B. J., \& Russell, A. F. (1996). Provisioning rules in cooperatively breeding long470 tailed tits Aegithalos caudatus: an experimental study. Proceedings of the Royal Society of 471 London B: Biological Sciences, 263(1366), 83-88.

472 Hatchwell, B. J. (1999). Investment strategies of breeders in avian cooperative breeding 473 systems. The American Naturalist, 154(2), 205-219.

474 Hatchwell, B.J., Russell, A.F., MacColl, A.D., Ross, D.J., Fowlie, M.K. and McGowan, A., 475 (2004). Helpers increase long-term but not short-term productivity in cooperatively breeding 476 long-tailed tits. Behavioral Ecology, 15(1), 1-10.

477 Heinsohn, R. G. (2004). Parental care, load-lightening, and costs. Ecology and evolution of 478 cooperative breeding in birds. Cambridge University Press, Cambridge, 67-80. 
479 Horváthová, T., Nakagawa, S., \& Uller, T. (2011). Strategic female reproductive investment in 480 response to male attractiveness in birds. Proceedings of the Royal Society of London B:

481 Biological Sciences, rspb20110663.

482 Hoyt, D. F. (1979). Practical methods of estimating volume and fresh weight of bird eggs. The 483 Auk, 73-77.

484 Jennions, M. D., Moller, A. P., \& Petrie, M. (2001). Sexually selected traits and adult survival: a 485 meta-analysis. Quarterly Review of Biology, 3-36.

486 Keller, L., \& Chapuisat, M. (2010). Eusociality and cooperation. eLS.

487 Kingma, S.A., Hall, M.L., Arriero, E. and Peters, A., 2010. Multiple benefits of cooperative 488 breeding in purple-crowned fairy-wrens: a consequence of fidelity?. Journal of Animal Ecology, $48979(4), 757-768$.

490 Koenig, W. D., \& Dickinson, J. L. (2004). Ecology and evolution of cooperative breeding in 491 birds. Cambridge University Press.

492 Koenig, W. D., Walters, E. L., \& Haydock, J. (2009). Helpers and egg investment in the 493 cooperatively breeding acorn woodpecker: testing the concealed helper effects 494 hypothesis. Behavioral ecology and sociobiology, 63(11), 1659-1665.

495 Koenig, W.D., Walters, E.L. and Haydock, J., 2011. Variable helper effects, ecological 496 conditions, and the evolution of cooperative breeding in the acorn woodpecker. The American 497 Naturalist, 178(2), 145-158. 
498 Krist, M. (2011). Egg size and offspring quality: a meta-analysis in birds. Biological 499 Reviews, 86(3), 692-716.

500 Lack, D. (1947). The significance of clutch-size. Ibis, 89(2), 302-352.

501 Lajeunesse, M. (2013). 13. Recovering Missing or Partial Data from Studies: A Survey of

502 Conversions and Imputations for Meta-analysis. Handbook of Meta-analysis in Ecology and 503 Evolution (pp. 195-206). Berlin, Boston: Princeton University Press.

504 Langmore, N. E., Bailey, L. D., Heinsohn, R. G., Russell, A. F., \& Kilner, R. M. (2016,

505 November). Egg size investment in superb fairy-wrens: helper effects are modulated by climate.

506 In Proc. R. Soc. B (Vol. 283, No. 1843, p. 20161875). The Royal Society.

507 Legge, S. (2000). Siblicide in the cooperatively breeding laughing kookaburra (Dacelo 508 novaeguineae). Behavioral Ecology and Sociobiology, 48(4), 293-302.

509 Lejeune, L., van de Pol, M., Cockburn, A., Louter, M., \& Brouwer, L. (2016). Male and female 510 helper effects on maternal investment and adult survival in red-winged fairy-wrens. Behavioral 511 Ecology, arw121.

512 Liebl, A. L., Nomano, F. Y., Browning, L. E., \& Russell, A. F. (2016). Experimental evidence 513 for fully additive care among male carers in the cooperatively breeding chestnut-crowned 514 babbler. Animal Behaviour, 115, 47-53.

515 Lindström, J. (1999) Early development and fitness in birds and mammals. Trends Ecol. 516 Evol. 14, 343-348. 
517 Lummaa, V., \& Clutton-Brock, T. (2002). Early development, survival and reproduction in

518 humans. Trends in Ecology \& Evolution, 17(3), 141-147.

519 Macaskill, P., Walter, S. D., \& Irwig, L. (2001). A comparison of methods to detect publication 520 bias in meta-analysis. Statistics in medicine, 20(4), 641-654.

521 Manica, L.T. and Marini, M.Â., 2012. Helpers at the nest of White-banded Tanager Neothraupis 522 fasciata benefit male breeders but do not increase reproductive success. Journal of Ornithology, 523 153(1),149-159.

524 Matsuura, K., \& Kobayashi, N. (2010). Termite queens adjust egg size according to colony 525 development. Behavioral Ecology, 21(5), 1018-1023.

526 McDonald, P. G., Kazem, A. J., \& Wright, J. (2009). Cooperative provisioning dynamics: fathers 527 and unrelated helpers show similar responses to manipulations of begging. Animal 528 Behaviour, 77(2), 369-376

529 Metcalfe, N. B., \& Monaghan, P. (2001). Compensation for a bad start: grow now, pay 530 later? Trends in ecology \& evolution, 16(5), 254-260.

531 Nakagawa, S., \& Santos, E. S. (2012). Methodological issues and advances in biological meta532 analysis. Evolutionary Ecology, 26(5), 1253-1274.

533 Paquet, M., Covas, R., Chastel, O., Parenteau, C., \& Doutrelant, C. (2013). Maternal effects in 534 relation to helper presence in the cooperatively breeding sociable weaver. PloS one, $8(3)$, 535 e59336. 
536 Paquet, M., Doutrelant, C., Hatchwell, B. J., Spottiswoode, C. N., \& Covas, R. (2015).

537 Antagonistic effect of helpers on breeding male and female survival in a cooperatively breeding

538 bird. Journal of Animal Ecology, 84(5), 1354-1362.

539 Pinheiro, J., Bates, D., DebRoy, S., Sarkar, D., and R Core Team (2015). nlme: Linear and

540 Nonlinear Mixed Effects Models. URL: http://CRAN.R-project.org/package=nlme

541 R Core Team (2015). R: A language and environment for statistical computing. R Foundation for

542 Statistical Computing, Vienna, Austria. https://www.R-project.org/.

543 RStudio Team (2015). RStudio: Integrated Development for R. RStudio, Inc., Boston, MA URL.

544 http://www.rstudio.com/.

545 Reyer, H.U., 1984. Investment and relatedness: a cost/benefit analysis of breeding and helping in

546 the pied kingfisher (Ceryle rudis). Animal Behaviour, 32(4), 1163-1178.

547 Rosenberg, M. S. (2010). A generalized formula for converting chi-square tests to effect sizes for 548 meta-analysis. PloS one, 5(4), e10059.

549 Rosenthal, R. (1994). Parametric measures of effect size. The handbook of research synthesis, $550 \quad 231-244$.

551 Russell, A. F., Langmore, N. E., Cockburn, A., Astheimer, L. B., \& Kilner, R. M. (2007).

552 Reduced egg investment can conceal helper effects in cooperatively breeding 553 birds. Science, 317(5840), 941-944. 
554 Russell, A. F., Langmore, N. E., Gardner, J. L., \& Kilner, R. M. (2008). Maternal investment

555 tactics in superb fairy-wrens. Proceedings of the Royal Society of London B: Biological

556 Sciences, 275(1630), 29-36.

557 Russell, A. F., \& Lummaa, V. (2009). Maternal effects in cooperative breeders: from

558 hymenopterans to humans. Philosophical Transactions of the Royal Society of London B:

559 Biological Sciences, 364(1520), 1143-1167.

560 Russell, A. F., Portelli, D. J., Russell, D. J. F., \& Barclay, H. (2010). Breeding ecology of the

561 chestnut-crowned babbler: a cooperative breeder in the desert. Emu, 110(4), 324-331.

562 Santos, E. S., \& Macedo, R. H. (2011). Load Lightening in Southern Lapwings: Group-Living

563 Mothers Lay Smaller Eggs than Pair-Living Mothers. Ethology, 117(6), 547-555.

564 Santos, E. S., \& Nakagawa, S. (2013). Breeding biology and variable mating system of a 565 population of introduced dunnocks (Prunella modularis) in New Zealand. PloS one, 8(7), 566 e69329.

567 Santos, S. J. D. (2016). Effects of group size on maternal allocation in a colonial cooperatively

568 breeding bird, the sociable weaver. Doctoral dissertation, University of Lisbon.

569 Savage, J. L., Russell, A. F., \& Johnstone, R. A. (2013). Intra-group relatedness affects parental 570 and helper investment rules in offspring care. Behavioral ecology and sociobiology, 1855-1865.

571 Savage, J. L., Russell, A. F., \& Johnstone, R. A. (2015). Maternal allocation in cooperative

572 breeders: should mothers match or compensate for expected helper contributions?. Animal 573 Behaviour, 102, 189-197. 
574 Sheldon, B. C. (2000). Differential allocation: tests, mechanisms and implications. Trends in

575 Ecology \& Evolution, 15(10), 397-402.

576 Shpigler, H., Tamarkin, M., Gruber, Y., Poleg, M., Siegel, A. J., \& Bloch, G. (2013). Social

577 influences on body size and developmental time in the bumblebee Bombus terrestris. Behavioral 578 Ecology and Sociobiology, 67(10), 1601-1612.

579 Smith, J. M. (1977). Parental investment: a prospective analysis. Animal Behaviour, 25, 1-9.

580 Stacey, P. B., \& Koenig, W. D. (1990). Cooperative breeding in birds: long term studies of 581 ecology and behaviour. Cambridge University Press.

582 Stearns, S. C. (1992). The evolution of life histories (Vol. 249). Oxford: Oxford University Press.

583 Taborsky, B., Skubic, E., \& Bruintjes, R. (2007). Mothers adjust egg size to helper number in a 584 cooperatively breeding cichlid. Behavioral Ecology, 18(4), 652-657.

585 Valencia, J., De La Cruz, C., Carranza, J., \& Mateos, C. (2006). Parents increase their parental 586 effort when aided by helpers in a cooperatively breeding bird. Animal Behaviour, 71(5), 10215871028.

588 Valencia, J., Mateos, C., Cruz, C., \& Carranza, J. (2016). Maternal allocation in eggs when 589 counting on helpers in a cooperatively breeding bird. Journal of Avian Biology.

590 Viechtbauer, W. (2010). Conducting meta-analyses in R with the metafor package. J Stat 591 Softw, 36(3), 1-48.

592 Villet, M. (1990). Qualitative relations of egg size, egg production and colony size in some 593 ponerine ants (Hymenoptera: Formicidae). Journal of Natural History, 24(5), 1321-1331. 
594 Visser, M. E., \& Lessells, C. M. (2001). The costs of egg production and incubation in great tits 595 (Parus major). Proceedings of the Royal Society of London B: Biological Sciences, 268(1473), $596 \quad 1271-1277$.

597 Williams, T. D. (2005). Mechanisms underlying the costs of egg production. Bioscience, 55(1), $598 \quad 39-48$.

599 Woxvold, I. A., Mulder, R. A., \& Magrath, M. J. (2006). Contributions to care vary with age, 600 sex, breeding status and group size in the cooperatively breeding apostlebird. Animal 601 Behaviour, 72(1), 63-73.

602 Wright, J. (1998). Helpers-at-the-nest have the same provisioning rule as parents: experimental 603 evidence from play-backs of chick begging. Behavioral Ecology and Sociobiology, 42(6), 423$604 \quad 429$ 